\title{
Quality Assurance of Adverse Drug Reaction Reporting and Monitoring by Clinical Pharmacists in a Tertiary Care Teaching Hospital
}

\author{
Himanshu Patel', Shobha Churi, Parthasarathi Gurumurthy, Ramesh Madhan \\ Department of Pharmacy Practice, JSS College of Pharmacy, Mysore, JSS University, Mysore 570015, INDIA.
}

\begin{abstract}
Background: Patient care services provided by clinical pharmacists should be evaluated periodically to ensure the quality of service. Objectives: This study was conducted to evaluate impact of standard operating procedures (SOPs) on quality of adverse drug reaction (ADR) reporting. Methods: The study was conducted by department of clinical pharmacy located at tertiary care teaching hospital. Quality assessment checklist was designed to assess the quality of ADR reports received through spontaneous reporting system and continuous monitoring. Based on score obtained from checklist ADR reports were graded at different quality levels. Quality of ADR reports was compared before and after implementation of SOP. Results: Before implementation of SOP, total of 71 ADRs were evaluated, out of which $40.85 \%$ of them were found to be good, whereas $39.43 \%$ of ADRs could be improved. However, only $11.26 \%$ of ADRs were found to be excellent and $8.46 \%$ of them needed an attention to improve it. After implementation of SOPs, 61 ADRs were evaluated, out of which $67.21 \%$ of them were found to be excellent and $16.39 \%$ of them were found to be good. Conclusion: The quality of ADR reporting and monitoring was found to be improved after implementation of SOPs. Ongoing quality monitoring may further help in maintaining standards and efficiency of ADR monitoring program at the study hospital.
\end{abstract}

Key words: Adverse drug reactions, Quality assurance, Standard operating procedures, Quality monitoring, Clinical Pharmacists.

\section{INTRODUCTION}

Adverse drug reaction (ADR) reporting is an essential key for monitoring safety of patients during clinical trials and post marketing surveillance. ${ }^{1}$ ADR reporting and monitoring programs encourage surveillance of adverse events following use of drugs and other medicinal products, documentation of such adverse events, promote the reporting of ADRs, provide structured approach for monitoring the safety of drugs in high risk populations and provide an ongoing opportunities to conduct educational programs for health care professionals in the hospital and community settings. ${ }^{2}$ ADR reporting programs are well developed at national and international levels in many developed countries. However, with the support of World Health Organization (WHO), many developing countries have also implemented drug safety surveillance programs in a last decade. ${ }^{3,4}$ Many countries have comprehensive ADR monitoring programs which include mechanisms for monitoring, detecting, evaluating, documenting, and reporting ADRs as well as intervening and providing educational feedback to prescribers, other health care professionals, and patients. Additionally, comprehensive ADR monitoring programs also focus on identifying problems leading to ADRs, planning for positive changes, and measuring the results of these changes. Positive outcomes resulting from an ADR monitoring program should be emphasized to support program growth and development. ${ }^{2,5}$

ADR monitoring programs possesses many benefits which includes but not limited to, providing an indirect measure of the qual-

Submitted date : 09/09/2015 Accepted date : 13/01/2016

DOI: 10.5530/ijopp.9.1.6

Address for

correspondence: Mr. Himanshu Patel

Lecturer and Clinical Pharmacist,

Department of Pharmacy Practice, JSS College of

Pharmacy, Mysore,

JSS University,

Mysore-570015, KA, INDIA

E-mail: himanshu051985@

yahoo.co.in

西


ity of pharmaceutical care through identification of preventable ADRs and anticipatory surveillance for high-risk drugs or patients, opportunity to develop risk assessment and management strategies at institutional level, assessing the safety of newer drug therapies and commonly used drugs in practice, quantification of ADRs, opportunity to educate health care professionals and patients about drug effects and increasing their level of awareness regarding ADRs. With established safety reporting system, we may also estimate economic burden of ADRs on patients and possible strategies can be developed to minimize the health care expenditures., ${ }^{2,-9}$

It is a responsibility and professional obligation of pharmacists to report ADRs. ${ }^{10}$ There are many published literature focusing and appreciating role of pharmacists in drug safety monitoring of patients. ${ }^{10-14}$ Pharmacists exert leadership in the development, maintenance, and ongoing evaluation of ADR programs in partnership with clinicians, nurses, patients and other health care professionals. While ADR reporting programs present these many benefits listed in above paragraph, it is equally important to monitor quality to ADR reporting. Poor quality of ADR report may showcase uncertain clinical condition, wrong understanding about pharmaceutical product, may challenge integrity of prescriber and negatively influences the patient care. Safety reporting guidance and/or having standard operating procedures (SOPs) at institutional level may help in ensuring compliance to ADR reporting processes which in turn generate good quality ADR reports.

\section{AIM OF THE STUDY}

This study was conducted to evaluate impact of SOPs on quality of ADR reporting and monitoring.

\section{MATERIALS AND METHODOLOGY}

This was a prospective study conducted by clinical pharmacy department located at tertiary care teaching hospital. This department of clinical pharmacy is a well-known drug information centre in the region and actively provides drugs and therapeutic information services in addition to other clinical pharmacy services like drug therapy review, patient medication counseling, and pharmacotherapy referrals for individualized drug therapy, patient referral for assessment of drug reactions and its management. The department of clinical pharmacy at the study hospital has been actively involved in ADR reporting and monitoring since 1997 and currently it is one of the peripheral centre for ADR monitoring under Pharmacovigilance Program of India (PvPi). Post graduate students of pharmacy practice (M.Pharm) and doctor of pharmacy (Pharm.D) students are considered as trainee clinical pharmacists/ward pharmacists at the study hospital and are usually posted to different medical departments on rotation basis to attend the ward rounds with the chief clinician, medical students and other health care professionals and to provide patient care services.

Trainee clinical pharmacists posted in to different wards and at pharmacy consultation area identified ADRs by interviewing patients and care takers, by reviewing patients' case notes and laboratory investigations and by discussing with clinicians. Identified ADRs were discussed by students with their pharmacy preceptors. After discussion all the ADRs were documented using ADR reporting and monitoring form of the department. Documented ADRs were also analyzed for its causality, severity, seriousness and predictability using WHO causality assessment scale, modified Hartwig \& Siegea scale, WHO seriousness scale for ADR, modified Shumock \& Thornton respectively. After completing documentation form, it was reviewed and authenticated by concerned clinician and concerned pharmacy preceptors. After authentication, ADR report was documented electronically in to department database by concerned trainee pharmacists.

Quality assessment panel was constituted with involvement of five members; four clinical pharmacists (2 senior level and 2 junior levels); one physician (senior level). In order to assess quality of ADRs, suitable quality assessment checklist was designed. A draft copy of quality assessment checklists were reviewed by all senior staff members $(n=4)$ of the department and 1 physician. Final copy of quality assessment checklists were prepared after incorporating given suggestions (Table 1). Based on the score obtained from checklist each evaluated ADR was categorized at different quality level; Excellent; Good; Can Improve; Should Improve. It was estimated to evaluate $50 \%$ of ADRs documented due to high volume of reports. However, no sample size calculation was performed to estimate the sample. The panel conducted a weekly audit of ADR reports to assess the quality. Disagreement among panelists if any, were sorted out by considering opinions of majority panelists. The quality was assessed for ADR reported for initial four months. After conduct of an audit each time, concerned students and/or pharmacy preceptors involved were invited to share discrepancies found and were requested to give their opinion about possible justification for discrepancies with the intention of quality improvement. Through initial four months' evaluation all the discrepancies reported during an audit were studied and they were considered to be factors leading to poor quality of services. Based on findings of initial four months, SOPs were developed for ADR reporting 
Table 1: Quality Assessment Checklist for ADR Reporting \& Monitoring

JSS MEDICAL COLLEGE HOSPITAL, MYSORE

Department of Clinical Pharmacy

Quality Assessment Checklist: Drug Information

Date of Documentation of Drug Information:

Name of Attending Pharmacist:

Date of Audit:

Reference No:

\begin{tabular}{ccc}
\hline \multicolumn{1}{c}{ Quality Assessment questionnaires } \\
\hline SI.No & Question & Yes \\
$\mathbf{1}$ & Was all required background information collected? \\
$\mathbf{2}$ & Was patient interviewed in detail specific to ADR? \\
$\mathbf{4}$ & Were appropriate resources referred to assess ADR? & Was suspected ADR discussed with concerned physician? \\
$\mathbf{5}$ & Was any appropriate suggestion given with regard to suspected ADR? & \\
$\mathbf{6}$ & Was suspected ADR assessed with respect to causality, severity, & \\
$\mathbf{7}$ & Seriousness, predictability and preventability? & \\
$\mathbf{8}$ & Was "ALERT CARD" given to the patient when needed or physician & insisted? \\
$\mathbf{9}$ & Was reported ADR and causality assessment reviewed by staff? & \\
\hline
\end{tabular}

Grade: $A$ (Excellent), B (Good), C (Can improve), D (Should improve)

Note: $A=8-10$ points, $B=6-7$ points, $C=4-5$ points, $D=3$ or less

Remarks:

Auditor:

Signature:

Note: Checked Yes carries 1 point. Checked No carries o point.

\section{Table 2: Standard Operating Procedure for ADR Reporting \& Monitoring}

Standard Operating Procedure for Adverse drug reaction reporting and monitoring

\subsection{Purpose and Scope}

1.1 This SOP enables the staff/students to adopt uniform approach in reporting, monitoring and managing the adverse drug reaction. 1.2 Adverse drug reaction reporting and monitoring is carried out for patients who are admitted and treated at JSS hospital, Mysore.

\subsection{Activity and Responsibility}

2.1 Identify ADR in patients during ward round participation/treatment chart review/receive ADR referral or report from the reporter. 2.2 Complete the suspected ADR notification form with reporter signature.

2.3 Collect all the relevant background information related to the ADR, patient's medical history and medication history.

2.4 Collect the information on patient's allergic status to any medications including other systems of medicine other than allopathy like ayurveda and homeopathy, and over- the counter-drugs and food.

2.5 Collect the information on suspected drug and specific information related to the suspected ADR like chronology of events, dechallenge and rechallenge.

2.6 Assess the causality of suspected ADR using the tools like Naranjo's Scale, WHO Scale and referring to appropriate drug information resources like primary resources (Journals), secondary resources (IDIS, Micromedex) and Tertiary resources (Text books). 2.7 If more than one drug is suspected in the ADR, assess the causality separately for each drug.

2.8 Assess the severity, predictability and preventability of the ADR using appropriate tools.

2.9 Get all the causality, severity, predictability and preventability assessments of the suspected ADR reviewed by the staff in-charge.

2.10 Counsel the patient about the ADR and its management.

2.11 Inform the patient in case any follow-up visits are required.

2.12 Inform the concerned reporter of the ADR about the causality assessment, management of ADR and methods to prevent the ADR, if the ADR is definitely/probably preventable.

2.13 Determine in case any additional information is required by the reporter regarding the ADR and provide the required information. 2.14 If the ADR meets the criteria for issuing an 'Alert Card', issue the alert cad to the patient and inform the concerned clinician about the issue of alert card.

2.15 Document the ADR including all assessment in the ADR documentation form.

2.16 File the completed ADR notification and documentation form maintained as per required in the ADR. 


\section{Table 3: Quality Assessment of Adverse drug reaction reporting before and after implementation of SOP}

\begin{tabular}{|c|c|c|c|c|}
\hline Month & Total ADR documented & Total ADR evaluated & Grade & Percentage \\
\hline July* & 33 & 17 & $3 \mathrm{~A}, 4 \mathrm{~B}, 6 \mathrm{C}, 4 \mathrm{D}$ & $\begin{array}{l}17.64 \% A, 23.53 \% B \\
35.30 \% C, 23.53 \% D\end{array}$ \\
\hline August* & 13 & 07 & $4 \mathrm{~B}, 2 \mathrm{C}, 1 \mathrm{D}$ & $\begin{array}{c}57.14 \% \mathrm{~B}, 28.58 \% \mathrm{C} \\
14.28 \% \mathrm{D}\end{array}$ \\
\hline September* & 19 & 10 & $1 \mathrm{~A}, 3 \mathrm{~B}, 5 \mathrm{C}, 1 \mathrm{D}$ & $\begin{array}{c}10 \% \mathrm{~A}, 30 \% \mathrm{~B}, 50 \% \mathrm{C} \\
10 \% \mathrm{D}\end{array}$ \\
\hline October* & 73 & 37 & $4 \mathrm{~A}, 18 \mathrm{~B}, 15 \mathrm{C}$ & $\begin{array}{c}10.81 \% \mathrm{~A}, 48.65 \% \mathrm{~B} \\
40.54 \% \mathrm{C}\end{array}$ \\
\hline Total & 138 & 71 & $8 A, 29 B, 28 C, 6 D$ & $\begin{array}{c}11.26 \% \mathrm{~A}, 40.85 \% \mathrm{~B} \\
39.43 \% \mathrm{C}, 8.46 \% \mathrm{D}\end{array}$ \\
\hline \multicolumn{5}{|c|}{ After implementation of SOP } \\
\hline November ${ }^{* *}$ & 45 & 23 & $17 \mathrm{~A}, 4 \mathrm{~B}, 2 \mathrm{NE}$ & $\begin{array}{c}73.92 \% \mathrm{~A}, 17.39 \% \mathrm{~B} \\
8.69 \% \mathrm{NE}\end{array}$ \\
\hline December** & 31 & 25 & $17 \mathrm{~A}, 3 \mathrm{~B}, 2 \mathrm{C}, 3 \mathrm{NE}$ & $\begin{array}{c}68.00 \% \mathrm{~A}, 12.00 \% \mathrm{~B} \\
8.00 \% \mathrm{C}, 12.00 \% \mathrm{D}\end{array}$ \\
\hline January** & 25 & 13 & $7 \mathrm{~A}, 3 \mathrm{~B}, 3 \mathrm{NE}$ & $\begin{array}{c}53.84 \% \mathrm{~A}, 23.07 \% \mathrm{~B} \\
23.07 \% \mathrm{NE}\end{array}$ \\
\hline Total & 101 & 61 & 41A, 10B, 2C, 8NE & $\begin{array}{l}67.21 \% \mathrm{~A}, 16.39 \% \mathrm{~B} \\
3.28 \% \mathrm{C}, 13.12 \% \mathrm{NE}\end{array}$ \\
\hline
\end{tabular}

Note: $A=$ Excellent, $B=G o o d, C=C a n$ improve, $D=$ should improve, NE=Not evaluated Months marked with * indicates study period when SOP was not implemented.

Months marked with **indicates study period when SOP was implemented.

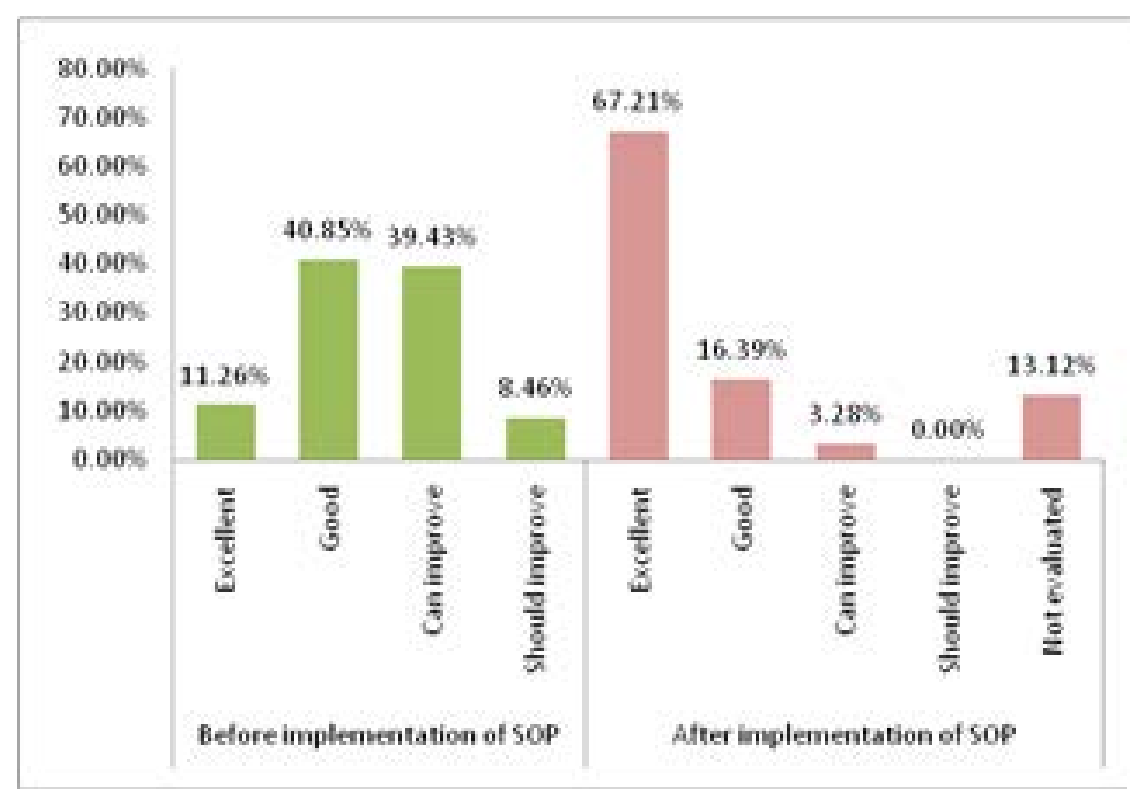

Figure 1: Quality of ADR reporting \& Monitoring before and after implementation of SOPs

and monitoring. A draft copy of SOP was provided to all the senior staff members of the department $(n=4)$ and one physician. There were few revisions of the draft SOPs after receiving feedback from SOP reviewers and then final SOP was prepared (Table 2). After designing SOPs, all post graduate students of pharmacy practice, Pharm.D students and staff members were educated about SOPs and they were advised to follow the same.
The SOPs was made available in department for general reference of all. Quality of ADRs were assessed for next 3 months after implementing SOPs. Quality of ADRs was measured in percentage at four different quality level; Excellent; Good; Can Improve; Should Improve. This study was approved by institutional ethical committee of JSS College of Pharmacy, Mysore, India. 


\section{RESULTS}

A total of 239 ADRs were reported during the study period of nine months. Of 239 ADRs, 138 ADRs were reported before implementation of SOPs and 101 ADRs were reported after implementation of SOPs. Before implementation of SOP, total of 71 ADRs were evaluated, out of which $40.85 \%$ of them were found to be good, whereas $39.43 \%$ of ADRs could be improved. However, only $11.26 \%$ of ADRs were found to be excellent and $8.46 \%$ of them needed an attention to improve it. Based on all the findings observed during initial evaluation, SOPs were designed and implemented.After implementation of SOPs, 61 ADRs were evaluated, out of which $67.21 \%$ of them were found to be excellent and $16.39 \%$ of them were found to be good. However, $3.28 \%(n=2)$ of them could be improved and $13.12 \%$ $(n=8)$ of them were not evaluated due to illegible hand writing. The detailed results of quality assessment are presented in Table 3. Figure 1 compares the quality of ADR reports before and after implementation of SOPs.

\section{DISCUSSION}

Quality assessment of clinical pharmacy services is usually performed to ensure the optimum standards of the services provided. Quality assurance programs further provides a confidence on efficiency and effectiveness of services to patient care and assures the competence of clinical pharmacists and clinical pharmacy department. Such programs also help to identify areas where improvement is needed.

Quality assessment of ADRs provide an opportunity to evaluate understanding of reporter about safety monitoring, process followed by different reporters to analyze the case, common resources used to study the drug reaction, to understand feedback of clinician to the pharmacist reported ADRs and to explore the potential impact of ADR reporting on patient care. In academic hospital setting, findings of such quality monitoring programs assist in developing training programs for pharmacy students, interns, pharmacy consultants and pharmacy preceptors. Also, it provides an opportunity to strengthen the policies and procedures of safety monitoring in the hospital. Guidance provided by national pharmacovigilance authorities of respective country and WHO collaborated Uppsala Monitoring Centre may help in developing educational programs for health care professionals of the hospital.

In the present study, audit was conducted as a method to assess the quality, as it is the most easy and suitable method for adoption. During initial assessment we found that nearly $40 \%$ of ADRs were rated as "Good". However, nearly $48 \%$ of ADRs needed an improve- ment. This seeks an attention to provide structured training to reporters and preceptors. The most common factors identified for ADR reports of sub optimal standards were lack of ADR specific back ground information, poorly assessed causality, incomplete documentation, lack of information on 'Alert Card" provided or not and poor attempts to follow the case for further feedback from clinician and to evaluate outcome of reaction. With discussion with concerned pharmacy preceptors we also found that many ADR reports were documented without authentication from their end. High work load of pharmacy preceptors and work shifts were other reasons for limited communication between trainee pharmacists and preceptors. Considering these factors, it was made mandatory under SOP to authenticate each reported case by pharmacy preceptor. After considering all the findings of initial evaluation, we structured SOPs and all the students and pharmacy preceptors were educated about the same. Post implementation of SOPs, we found majority of ADRs were rated as "Excellent" and "Good". Interestingly, none of the ADR report needed significant attention. However, we could not evaluate few $(n=8)$ ADRs due to illegible hand writing. Concerned reporter and preceptors were informed about the same with the corrective actions. We thoroughly reviewed all the factors led to poor quality reports during initial evaluation and all of them were found to be improved.

A study conducted by Bandekar MS and his colleagues evaluated quality of ADR reporting forms of various countries against self-designed quality criteria for ADR reporting and did not evaluate quality of actual ADR reports received by spontaneous reporting system. ${ }^{15} \mathrm{~A}$ study conducted by Gedde-Dahl A et al. studied the quality between pharmacist reported ADRs and clinician reported ADRs and they found that pharmacist reported ADRs were more informative than clinicians. ${ }^{16} \mathrm{~A}$ study conducted by Marco Tuccori et al performed quality check of ADRs reports through QADRA algorithm which was designed by their study team to assess causality of drug reactions in a better way. ${ }^{17} \mathrm{~A}$ study conducted by David A Dorr and his colleagues evaluated quality, accuracy and completeness of imatinib induced serious adverse events submitted to institutional review board and they had used validated clinical data fields and causality assessment instrument. David A Dorr concluded that structured approach of reporting adverse events improves quality of adverse event reports. ${ }^{18}$ Our study results also conveyed the same and highlighted importance of standardized approach for reporting adverse drug reactions. To our knowledge this is the first study evaluating quality of spontaneously reported ADRs through specially designed quality checklist at academic level in India. 


\section{CONCLUSION}

Quality monitoring program of ADRs was found to be very useful to identify areas of improvement. The quality of ADR reporting and monitoring was found to be improved after implementation of SOPs. This study shows positive impact of structured approach on quality of drug safety monitoring. Ongoing quality monitoring may further help in maintaining standards and efficiency of ADR monitoring program at the study hospital.

\section{ACKNOWLEDGMENTS}

Authors thank to Dr. M.G.Narahari, Associate Professor, Department of General Medicine, JSS Hospital, Mysore for his support and encouragement towards quality monitoring program.

Source of funding: No funding was received for this study.

\section{REFERENCES}

1. Vlahović-Palčevski V, Mentzer D. Postmarketing surveillance. Handb Exp Pharmacol. 2011; 205:339-51.

2. ASHP Guidelines on Adverse Drug Reaction Monitoring and Reporting, Medication Misadventures-Guidelines. 211-13.

3. Olsson S, Pal SN, Stergachis A, Couper M. Pharmacovigilance activities in 55 low- and middle-income countries: a questionnaire-based analysis, Drug Saf. 2010;33(8):689-703.

4. Aagaard L, Strandell J, Melskens L, Petersen PS, Holme Hansen E. Global patterns of adverse drug reactions over a decade: analyses of spontaneous reports to Vigi Base ${ }^{\mathrm{TM}}$. Drug Saf. 2012;35(12):1171-82.

5. Murphy BM, Frigo LC. Development, implementation, and results of a successful multidisciplinary adverse drug reaction reporting program in a university teaching hospital. Hosp Pharm. 1993;28(12):1199-204.
6. Ramesh M, Pandit J, Parthasarathi G. Adverse drug reactions in a south Indian hospital--their severity and cost involved. Pharmacoepidemiol. Drug Saf. 2003;12(8):687-92.

7. Bordet R, Gautier S, Le Louet H, Dupuis B, Caron J. Analysis of the direct cost of adverse drug reactions in hospitalised patients. Eur $\mathrm{J}$ ClinPharmacol. 2001;56(12):935-41.

8. Owenby RK, Brown JN, Kemp DW. Evaluation of pharmacy services in emergency departments of Veterans Affairs Medical Centers. Am J Health Syst Pharm. 2015; 72(17Suppl2):S110-4.

9. Baniasadi s, Fahimi F and Shalviri G. Developing an Adverse Drug Reaction Reporting System at a Teaching Hospital. Basic \& Clinical Pharmacology \& Toxicology 2008;102(4):408-11.

10. Baniasadi S et al, Increasing the Number of Adverse Drug Reactions Reporting: the Role of Clinical Pharmacy Residents. Iran J Pharm Res. 2014;13(1):291-7.

11. Marie Gabrielle Guédat et al, Pharmacovigilance and clinical pharmacy at the hospital:assessment and prospects after 9 years of experience. Therapy. 673(2012):243-50.

12. Sánchez I, Amador C, Plaza JC, Correa G, Amador R, Assessment of an active pharmacovigilance system carried out by a pharmacist. Rev Med Chil. 2014; 142(8):998-1005.

13. Ratz Y, Shafir I, Berkovitch S, Sharristh M, Jacoby M, Kozer E et al.The Importance of the Pharmacist in Reporting Adverse Drug Reactions in the Emergency Department. J Clin Pharmacol. 2010;50(10):1217-21.

14. Glassman P. Making Health Care Safer II ; An Updated Critical Analysis of the Evidence for Patient Safety Practices. Clinical Pharmacist's Role in Preventing Adverse Drug Events: Brief Update Review. Rockville (MD): Agency for Healthcare Research and Quality (US); 2013 Mar. (Evidence Reports/ Technology Assessments, No. 211.).

15. Bandekar MS, Anwikar SR, Kshirsagar NA. Quality check of spontaneous adverse drug reaction reporting forms of different countries. Pharmacoepidemiol Drug Saf. 2010; 19(11):1181-5.

16. Gedde-Dahl A, Harg P, Stenberg-Nilsen H, Buajordet M, Granas AG, Horn AM. Characteristics and quality of adverse drug reaction reports by pharmacists in Norway. Pharmacoepidemiol Drug Saf. 2007 Sep; 16(9):999-1005.

17. Tuccori $\mathrm{M}$ et al. Quality of Adverse Drug Reaction (QADRA) reports: an algorithm to appraise the efficiency of spontaneous reporting systems in pharmacovigilance, Journal of Public Health. 2013;21(4):365-72.

18. Dorr DA, Burdon R, West DP, Lagman J, Georgopoulos C, Belknap SM et al, Quality of Reporting of Serious Adverse Drug Events to an Institutional Review Board, A Case Study with the Novel Cancer Agent, Imatinib Mesylate. Clin Cancer Res. 2009;15(11):3850-5. 